\title{
Stability of the hcp phase and temperature variation of the axial ratio of iron near Earth-core conditions
}

\author{
P Modak ${ }^{1}$, A K Verma ${ }^{1}$, R S Rao ${ }^{1}$, B K Godwal ${ }^{2}$, L Stixrude ${ }^{3}$ and \\ R Jeanloz ${ }^{2}$ \\ ${ }^{1}$ High Pressure Physics Division, Bhabha Atomic Research Centre, Trombay, 400085, India \\ 2 Department of Earth and Planetary Science, University of California, Berkeley, CA 94720-4767, \\ USA \\ ${ }^{3}$ Department of Geological Sciences, University of Michigan, 425 E University Avenue, Ann \\ Arbor, MI 48109-1063, USA
}

Received 29 August 2006, in final form 10 November 2006

Published 7 December 2006

Online at stacks.iop.org/JPhysCM/19/016208

\begin{abstract}
We theoretically document the stability of hcp iron for pressure-temperature conditions of the Earth's inner core by separately computing the electronic and phonon contributions to the free energy. These pseudopotential-based quasiharmonic calculations reveal that the hcp phase remains stable compared to bcc and that the $c / a$ ratio of lattice parameters exhibits only a modest temperature dependence at inner-core conditions.
\end{abstract}

\section{Introduction}

The high-pressure, high-temperature properties of iron have enormous importance in the fields of geoscience, seismology and cosmochemistry because it is one of the most abundant heavy elements in the Solar System. In spite of significant developments in experimental techniques (for example, laser-heated diamond-anvil cells), it has not been possible to study iron, the most abundant element in Earth's solid inner core, at pressure-temperature conditions near $330 \mathrm{GPa}$ and $6000 \mathrm{~K}$. There has been renewed interest in the structural properties of iron at these conditions due to the observation that compressional seismic waves travel faster along the Earth's rotation axis than in the equatorial plane [1], implying that the inner core is anisotropic. Several models have been proposed to explain this anisotropy, for example in terms of preferred orientation of crystals which might have happened during growth of the inner core [2-4] or by solid-state deformation [5]. The forces responsible for deformation are unknown, although several mechanisms have been proposed [6, 7]. Hence, understanding the origin of the anisotropy in the inner core with the help of the high-pressure, high-temperature properties of iron may shed light on the dynamics and evolution of our planet's deepest interior.

Unfortunately, the exact composition of the core and the crystal structure of its predominant iron component are controversial [8, 9]. Experimental approaches being limited, an alternative approach is to simulate iron and determine its structural and elastic properties 
at Earth-core conditions. There have been experimental [10] as well as theoretical attempts to do this [11-14], some of which used first-principles methods applied to high pressures and temperatures [12-14], but their conclusions are at variance with each other. Stixrude and Cohen [4] conclude that in the Earth's core iron has the hcp structure, as an aligned aggregate of crystals can satisfactorily explain the observed seismic travel-time anomaly, and they found the bcc phase to be dynamically unstable $[15,16]$. They also noted that a cylindrically averaged aggregate of fcc crystals disagrees with seismological observations, with travel-time anomalies at the pole $(+5.8 \mathrm{~s})$ and equator $(+2.2 \mathrm{~s})$ being overestimated. This apparently rules out fcc as a candidate for the crystal structure of iron in the Earth's core.

However, molecular dynamics (MD) studies using embedded-atom model potentials predict the stability of the bcc over the hcp phase of pure iron [13]. Vocadlo et al [11], employing ab initio molecular dynamics, predicted the mechanical stability of the bcc phase of pure iron; but hcp would still be thermodynamically stable, and have the lowest Gibbs free energy. They also illustrated that addition of a few per cent light elements such as sulfur, oxygen and silicon could tend to favour the bcc phase in the Earth's core conditions.

Assuming the hcp structure of iron, Wasserman et al [17], Steinle-Neumann et al [14] and Gannarelli et al [18] studied the temperature variation of the $c / a$ ratio, using the particle-in-acell (PIC) method, but obtained different results. Wasserman et al [17] used the combined tight-binding total energy (TBTE) method with the cell model of the vibrational partition function and predicted a rapid increase of $c / a$ with temperature. Steinle-Neumann et al [14], using the pseudopotential method with PIC found that $c / a$ grows with temperature, from about 1.6 near $0 \mathrm{~K}$ to about 1.7 at $6000 \mathrm{~K}$, and found agreement with the variation obtained by Wasserman et al [17]. However, Gannarelli et al [18] obtained a lesser increase over the same temperature range. This discrepancy is surprising, as similar methods have been employed in these calculations. In the later calculation, Gannarelli et al [19] used the frozen phonon method to calculate harmonic phonon spectra and then added the anharmonic contributions, obtaining them either by thermodynamic integrations or by ab initio MD simulations. In MD simulations, the equilibrium axial ratio was determined from calculation of the stress difference $\sigma_{33}-\sigma_{11}$, which disappears for the equilibrium value of $c / a$. They obtained a larger increase of $c / a$ with temperature as compared with their previous PIC results, but still smaller than that found by Steinle-Neumann et al [14]. They also found that the geometric mean frequency differs by a constant factor compared to their PIC results.

All these controversies regarding the temperature variation of $c / a$ raise questions about the applicability of the PIC method to iron at Earth-core conditions, although it was found by Gulseren and Cohen [20] to give adequate results for thermoelastic properties of bcc Ta for pressures of $400 \mathrm{GPa}$ and temperatures of $10000 \mathrm{~K}$. The essence of the PIC method is that correlations between the vibrational displacements of different atoms are neglected, so each atom is treated as vibrating independently of the others and hence doubts have been raised about the applicability of this method [21]. However, Sha and Cohen [22] recently studied the lattice dynamics and thermodynamics of $\varepsilon$-iron at high pressures and high temperatures, using both the linear-response full-potential linear muffin-tin orbital (LMTO) method and the tightbinding-model based PIC. They found that both methods give comparable results for various quantities like geometric-mean frequency, the Helmholtz free energy and the axial ratio for the stable hcp structure of $\mathrm{Fe}$ as a function of pressure and temperature. However, they obtained a smaller temperature variation of $c / a$. According to them, the large temperature variation of $c / a$ as obtained in some earlier PIC calculations is due to errors in computing the special directional integrations. They also noted that the PIC method is not applicable close to a lattice instability.

In the present study, we employ conventional high-temperature solid-state theory to obtain the free energies of different crystal structures of iron. We evaluate the free-energy of the 
system, including contributions from the cold lattice, harmonic lattice vibrations, anharmonic effects and thermal excitation of electrons. The electronic-band structure is used to obtain the electronic contribution. We have used ab initio phonon calculations ${ }^{4}$ and the Debye model [23] for evaluating the lattice contribution to the free energy. The anharmonic-phonon and thermal electronic contributions are included phenomenologically [23]. We also estimated separately the thermal electronic excitation using temperature-dependent density-functional theory [24]. The validity of our approach is supported by the agreement of our computed phonon density of states with experiments at $112 \mathrm{GPa}$ [25]. Support for our use of the pseudopotential approach is provided by first-principles MD simulations of the viscosity of molten iron at the conditions of the Earth's core [26]. A similar approach has been used by Sha and Cohen [27] in their recent study on the lattice dynamics and thermodynamics of iron under pressure using first-principles linear-response theory. In their work on the thermal equation of state of ferromagnetic bcc iron they used the quasi-harmonic approximation with an all-electron method.

We have recently calculated the relative phase stabilities of the bcc, hcp, dhcp and orthorhombic $(\mathrm{Pbcm})$ phases of Fe using an accurate ultrasoft pseudopotential [28]. We found from these studies that at $0 \mathrm{~K}$ the hcp phase is the most stable phase among them, at least up to a pressure of $300 \mathrm{GPa}$. Our computed energy differences with respect to hcp, are of the order of $5 \mathrm{mRyd} /$ atom for the orthorhombic phase, and of the order of 3-4 mRyd/atom for fcc and dhcp [28]. Also, our geometrical optimization for the orthorhombic structure showed that the structure is elastically unstable. In those calculations, we did not compute the vibrational free energy to determine phase stability, but based on a rough estimate argued that it would not change our conclusions.

In the present work, we have carried out calculations including the thermal-vibrational contribution to the free energy using the phonon density of states (PDOS) obtained from $a b$ initio phonon frequency calculations for $\mathrm{Fe}$ at a volume corresponding to pressures of the Earth's inner core. We also evaluated the thermal contribution to the free energy within the Debye model, because for some structures such as orthorhombic and bcc, the ab initio phonon frequency calculations yielded imaginary frequencies at some q-points in the Brillouin zone (BZ). We have undertaken this exercise to establish the reliability with which the thermal contribution to the free energy can be determined. In view of the importance of the $c / a$ variation with temperature for hcp iron, and because this is potentially an experimentally measurable property at elevated pressures and temperatures, we have calculated it from freeenergy minimization at each temperature. Our calculations confirm the stability of hcp iron, and we compare the predicted temperature variation of $c / a$ with the results of theoretical calculations and experimental data. In the present work, the PWSCF numerical code (see footnote 4) was used to find the electronic and harmonic phonon contributions to the free energy.

\section{Details of calculations}

The Helmholtz free energy at a given volume $V$ and temperature $T$ is written as

$$
F(V, T)=E_{0}+F_{\mathrm{H}}(T)+F_{\mathrm{AE}}(T) .
$$

Here $E_{0}$ is the internal energy of the static lattice and $F_{\mathrm{H}}(T)$ and $F_{\mathrm{AE}}(T)$ are the thermal contributions at a given volume $V$ to the Helmholtz free energy from harmonic lattice vibrations of the ions and from the combined contribution of electronic excitations and anharmonic phonons, respectively.

4 For PWSCF visit http://www.pwscf.org. 
We evaluated the thermal contribution to the free energy using the phonon density of states, $g(\omega)$, obtained from $a b$ initio phonon calculations within the quasi-harmonic approximation using the PWSCF code (see footnote 4):

$$
F_{\mathrm{H}}(T)=\int_{0}^{\omega_{\max }}\left\{\hbar \omega / 2+k_{\mathrm{B}} T \ln \left[1-\exp \left(-\hbar \omega / k_{\mathrm{B}} T\right)\right\} g(\omega) \mathrm{d} \omega .\right.
$$

We also calculated the free energy using Wallace's prescription [23], because for some structures one cannot evaluate it using the phonon spectrum due to the structures being unstable at $0 \mathrm{~K}$. The total energy calculations are based on density-functional theory (DFT), and the phonon calculations on density-functional perturbation theory [29].

The interactions between ions and valence electrons are described using ultrasoft pseudopotentials, with a 25 Ryd plane-wave energy cut-off and a 300 Ryd cut-off in the expansion of the augmentation charges. For the BZ integration, we used a $20 \times 20 \times 10 \mathbf{k}$ point mesh for the hcp structure and a $15 \times 15 \times 15 \mathbf{k}$-point mesh for the fcc structure. For dhcp and orthorhombic structures we used comparable k-point meshes. Phonon dynamical matrices are computed on a uniform $5 \times 5 \times 3$ and $4 \times 4 \times 4$ grid of q-points in the BZ, including the $\Gamma$ point, for hcp and fcc structures, respectively. Using Fourier interpolation, we constructed and diagonalized the dynamical matrices in a denser grid, yielding a large number of phonon frequencies for a particular volume, and then used them to evaluate the phonon density of states $g(\omega)$ and Helmholtz free energy at a given volume $V$ as a function of temperature.

For the regions in which the dhcp, orthorhombic and bcc structures are vibrationally unstable, we calculated the lattice free energy using Wallace's prescription [23], because for these structures the phonon spectrum includes imaginary frequencies due to the structure being unstable at $0 \mathrm{~K}$. We assume a Debye model with $\theta_{\mathrm{D}}$ obtained from the calculated bulk modulus (B) (neglecting shear stress) and the density $(\rho)$ of the solid to obtain the thermal lattice free energy for dhcp, orthorhombic and bcc phases.

The phonon contributions are treated in the high-temperature regime in accordance with Wallace [23]. For the harmonic part, the contribution to the free energy is

$$
F_{\mathrm{H}}(T)=-3 k_{\mathrm{B}} T\left[\ln \left(T / \theta_{0}\right)-(1 / 40)\left(\theta_{2} / T\right)^{2}+\cdots\right]
$$

with $\theta_{0}$ and $\theta_{2}$ treated as phenomenological parameters:

$$
\theta_{0}=\theta_{\mathrm{D}} \mathrm{e}^{-1 / 3}
$$

and

$$
\theta_{2} \approx \theta_{\mathrm{D}}
$$

where

$$
\theta_{\mathrm{D}}=\left(\hbar v_{\mathrm{s}} / k_{\mathrm{B}}\right)\left(6 \pi^{2} n\right)^{1 / 3}
$$

$v_{\mathrm{s}}=(B / \rho)^{1 / 2}$ is the average sound velocity and $n$ is the number density of the atoms.

With inclusion of lattice-phonon contributions for all the structures using either equations (2) or (3), we evaluate $F_{\mathrm{AE}}$ in equation (1) to obtain the total free energy at a given volume $V$ and temperature $T$ following Wallace [23]

$$
F_{\mathrm{AE}}(T)=-\Gamma T^{2} .
$$

Here $\Gamma$ is a phenomenological parameter determined from available specific heat data on iron at high temperatures and pressures $[6,23,30]$. 


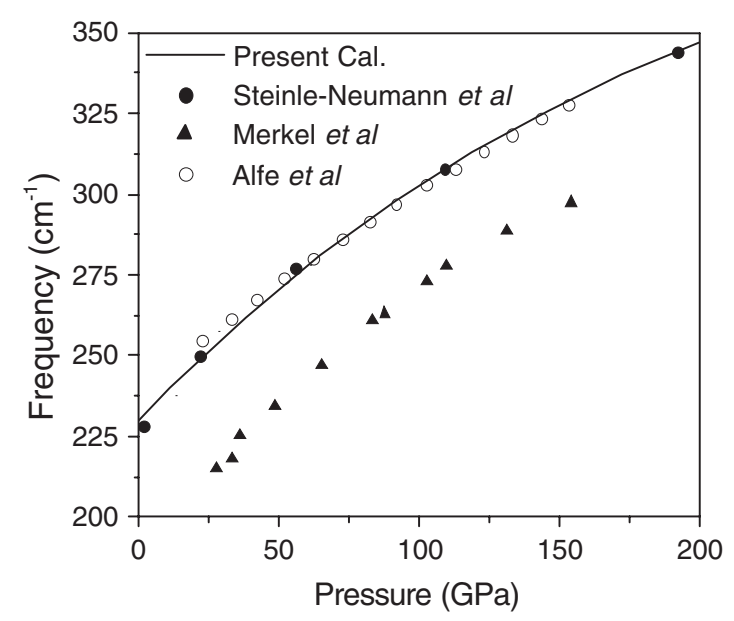

Figure 1. Pressure variation of the $E_{2 g}$ Raman phonon frequency of hcp iron. The solid line is a second-order polynomial fit to our calculated frequencies; solid and open circles are, respectively, Steinle-Neumann et al's [31] and Alfe et al's [32] theoretical results. Solid triangles are the experimental data of Merkel et al [30].

\section{Results}

In our earlier work [28] we had compared the calculated equilibrium properties and ambientpressure phonon frequencies with the available experimental data and other theoretical calculations, and found reasonable agreement. Here we focus on the high-pressure, hightemperature properties of iron. To study the phase stability of iron at moderate and high pressures and at high temperatures, we have calculated the $0 \mathrm{~K}$ total energies for hcp, dhcp, fcc, bcc and orthorhombic phases at different compressions, and the full phonon spectrum for hcp and fcc phases for volumes corresponding to pressures of 100 and $400 \mathrm{GPa}$ at zero temperature. To illustrate the reliability of our calculations, we compare in figure 1 our computed Ramanactive $\mathrm{E}_{2 \mathrm{~g}}$-mode phonon frequency of the non-magnetic hcp phase under compression with the results of other calculations [31,32] and experiments [30]. All the theoretical results agree with each other, and the predicted hardening under pressure is similar to that of the experiments, though the frequency is shifted upward.

The calculated PDOS for hcp Fe is in reasonable agreement with the experimental data of Mao et al [25] (figure 2). It is clear that both spectra are qualitatively similar, although the experimental frequency distribution is slightly spread out. A mismatch between theory and experiment on the scale shown in figures 1 and 2 is not unusual in first-principles estimates of phonon spectra [33].

Our free-energy calculations for different phases show that the hcp phase is more stable than the fcc phase at $100 \mathrm{GPa}$ pressure up to $2500 \mathrm{~K}$, which is consistent with existing knowledge [34] of the phase diagram of iron at moderate pressure-temperature conditions (see figure 3). Our results, which explicitly include thermal contributions to the free energy, are more reliable than earlier approaches that considered the entropy term only approximately [35]. From figure 3, it is seen that our calculated hcp to fcc transition temperature at $100 \mathrm{GPa}$ pressure is close to that of Boehler's [36] diamond anvil cell (DAC) measurements.

For the Debye model, the calculated bulk moduli were used to obtain the Debye temperature $\left(\theta_{\mathrm{D}}\right)$ 1723, 1656 and $1463 \mathrm{GPa}$, for dhcp, orthorhombic and bcc phases, giving $\theta_{\mathrm{D}}$ of 1737,1702 and $1601 \mathrm{~K}$, respectively, at a volume of 41.44 (au) ${ }^{3}$ per atom. Using 


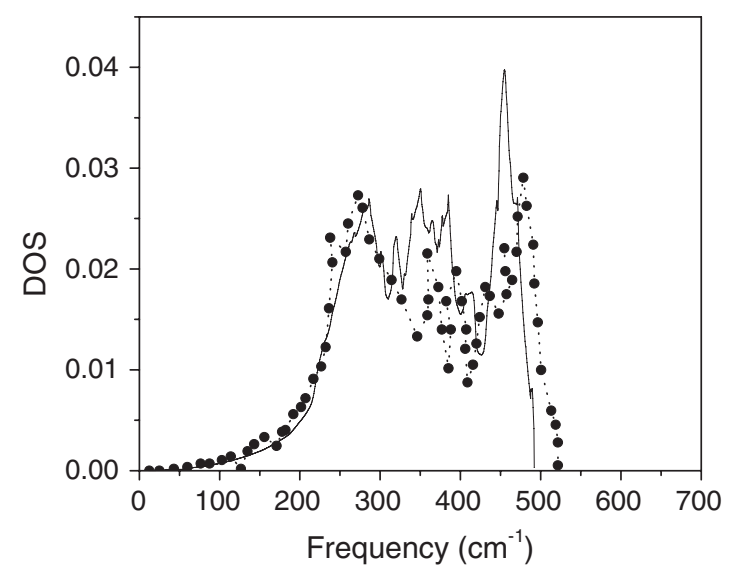

Figure 2. Comparison of the experimental phonon density of states (PDOS) measured at $112 \mathrm{GPa}$ pressure (points) (see footnote 4) with our theoretical estimate (line). Experimental PDOS is in arbitrary units with large uncertainties in the high-frequency region.

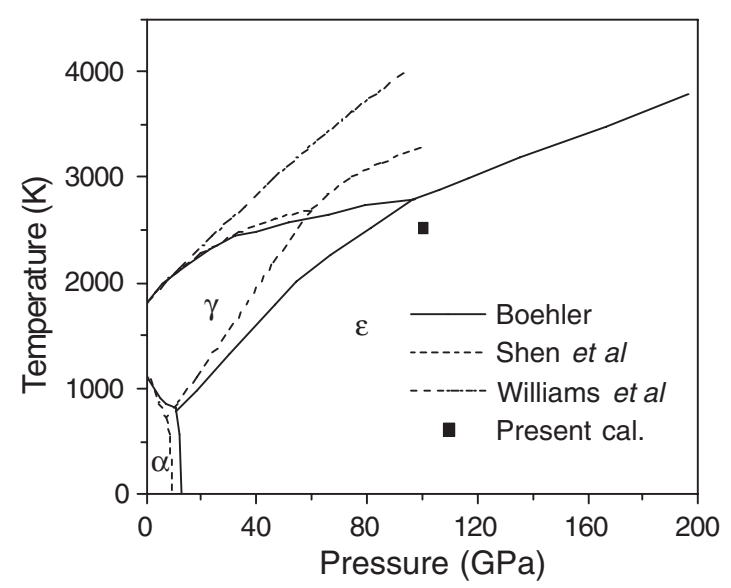

Figure 3. Iron phase diagram. Our calculated hep $(\varepsilon)$ to fcc $(\gamma)$ transition temperature at $100 \mathrm{GPa}$ pressure is shown by the filled square $(\square)$ along with measured solid-solid phase boundaries and melt lines obtained from DAC experiments by Boehler [36], Shen et al [37] and Williams et al [38].

$\theta_{\mathrm{D}}$ for the value of $\theta_{2}$ in Wallace's prescription [23], we calculated the phonon contribution to the free energy. We also estimated the contribution of the electronic excitation to the free energy from temperature-dependent density-functional theory [24]. For inclusion of the anharmonic contribution we followed Wallace [23], and combined it with the free-electron model for electronic excitation as shown in equation (7).

The variation of the total free energy differences between hcp and other phases with temperature is shown in figure 4 for a volume compression corresponding to a pressure of $400 \mathrm{GPa}$ for the hcp phase. At this pressure, the hcp phase is lower in energy, at least up to $6000 \mathrm{~K}$. Though the energy difference between hcp and bcc phases decreases with temperature at this pressure, it is still of the order of $15 \mathrm{mRyd}$ at $6000 \mathrm{~K}$. This is quite a large energy difference, hence our calculations rule out the possibility of bcc iron being stable at Earth-core conditions in accord with earlier calculations by Stixrude et al [16]. The temperature-dependent density-functional electronic excitation contribution differs from the combined anharmonic and 


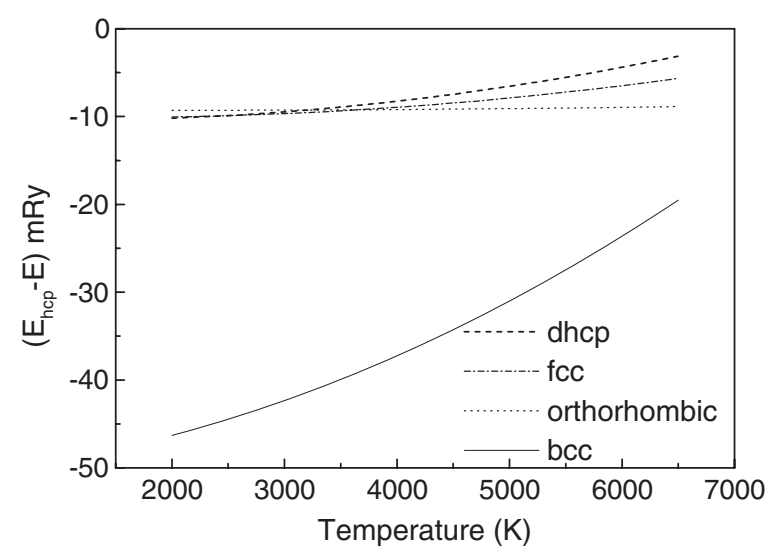

Figure 4. Thermal variations of free energy differences between hcp and other phases of iron at a volume corresponding to a $0 \mathrm{~K}$ pressure of $400 \mathrm{GPa}$ in the hcp phase (calculated within the Debye model).

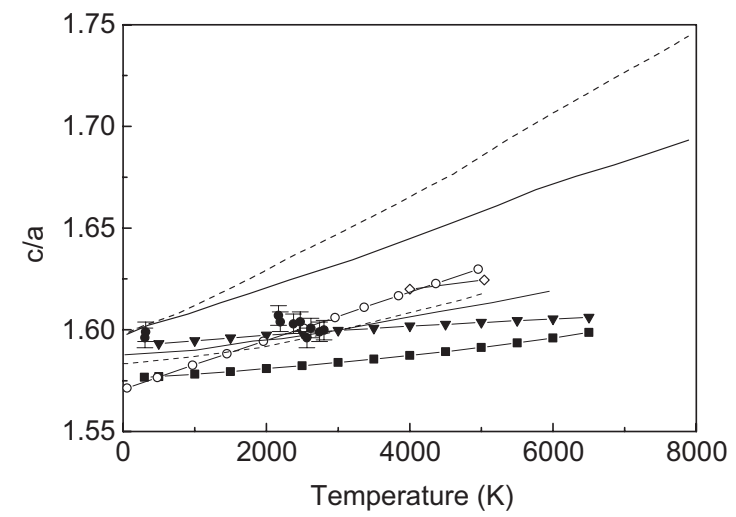

Figure 5. Calculated equilibrium axial ratio as a function of temperature for different volumes. For the present work, atomic volumes are chosen as $7.11 \AA^{3}$ (solid square) and $6.14 \AA^{3}$ (down triangle). Gannarelli et al's [19] data are for volumes of $7.5 \AA^{3}$ (light dotted curve), $6.97 \AA^{3}$ (light solid curve) and $a b$ initio MD calculations (open diamonds) at $6.97 \AA^{3}$. For Steinle-Neumann et al [14], volumes are $6.81 \AA^{3}$ (heavy solid curve) and $7.11 \AA^{3}$ (heavy dashed curve). Also shown are values from X-ray diffraction measurements of Ma et al [10] at $7.73 \AA^{3}$ /atom (solid circles with error bars) and MD results based on an embedded atom potential of Belonoshko et al [13] (open circles) at $7.2 \AA^{3}$.

free electron excitation by $5 \%$. We expect that a more rigorous treatment of the anharmonic contribution cannot make up for this difference.

Our calculations support the conclusion that the hcp phase of iron is stable at the pressures of the Earths core, in agreement with the results of Vocadlo et al [11], who employed ab initio molecular dynamic simulations to evaluate the free energies of the hcp and bcc phases. This agreement helps justify our phenomenological handling of the anharmonic-phonon contribution to the free energy.

In order to study the temperature variation of the $c / a$ ratio, we calculated the phonon density of states at the volume corresponding to Earth-core pressure and optimized $c / a$ based on the free energy calculated for temperatures between 300 and $6500 \mathrm{~K}$. Figure 5 shows the 
resulting temperature variation of the $c / a$ ratio for hep iron at volumes corresponding to a pressure of $400 \mathrm{GPa}$.

In figure 5 , we compare the $c / a$ variation with temperature with experimental data and with results from other theoretical calculations. We find that there is a small increase of the $c / a$ ratio of hcp iron with temperature at high pressure. At zero temperature, all the predictions for $c / a$ values more or less match. The experimental data in figure 5 initially shows an increasing trend with temperature, but at higher temperatures a slight decrease is observed. However, this decrease is not found in the theoretical prediction, and may be due to assumptions in the calculations (e.g. constant volume) or to uncertainties in the experiments. At higher temperatures, our values are lower than those of any other estimates. We note from the figure that our results show better agreement with Gannarelli et al [19] than do earlier calculations. We have also compared our estimates with recent results on $c / a$ variation with temperature by Sha and Cohen [22] and find similar agreement. By neglecting the anharmonic contribution and estimating electronic excitation from temperature-dependent density-functional theory, the $c / a$ values changed roughly by $0.04 \%$. Thus we note that either the anharmonic contribution is negligible or it lacks a rigorous treatment. These possibilities can perhaps best be resolved by first-principles MD simulations with a large number of atoms.

\section{Conclusions}

We have revisited the question of the stability of iron phases at Earth-core conditions, employing an approach that separately evaluates contributions from electronic free energy, harmonic phonons and anharmonic effects, and our free-energy calculations confirm that the stable phase has the hcp structure. To address the temperature variation of the $c / a$ ratio in the hcp phase at high pressure, we have evaluated the optimum $c / a$ ratio obtained by minimizing our calculated free energy at various temperatures. We find a small increase of $c / a$ with temperature, and our results show good agreement with those of Gannarelli et al [19] and recent results of Sha and Cohen [22]. In view of the limitations arising from use of the PIC model, as far as phonon correlations are concerned, and our inability to adequately include anharmonic contributions to the free energy calculations, it is desired to have detailed ab initio MD simulations with large numbers of atoms in the simulation cell at Earth-core conditions. There is also an urgent need to have more experimental $c / a$ data at different pressures as a function of temperature.

\section{Acknowledgments}

PM, AKV, RSR and BKG acknowledge helpful discussions with Dr D M Gaitonde.

\section{References}

[1] Morelli A, Dziewonski A M and Woodhouse J H 1986 Geophys. Res. Lett. 131545

[2] Bergman M I 1997 Nature 38960

[3] Karato S I 1993 Science 2621708

[4] Stixrude L and Cohen R E 1995 Science 2671972

[5] Buffett A B and Bloxham J 2000 Geophys. Res. Lett. 244001

[6] Jeanloz R and Wenk H R 1988 Geophys. Res. Lett. 1572

[7] Yoshida S, Sumita I and Kumazawa M 1996 J. Geophys. Res. 10128085

[8] Buffett A B, Huppert H E, Lister J R and Woods A W 1996 J. Geophys. Res. 1017989

[9] Mao H K, Wu Y, Chen L C and Shu J F 1990 J. Geophys. Res. 9521737

[10] Ma Y, Somayazulu M, Shen G, Mao H K, Shu J and Hemley R 2004 Phys. Earth Planet Inter. 143/144 455 
[11] Vocadlo L, Alfe D, Gillan M J, Wood I G, Brodholt J P and Price G D 2003 Nature 424536

[12] Soderlind P and Moriarty J A 1996 Phys. Rev. B 5314063

[13] Belonoshko A B, Ahuja R and Johansson B 2003 Nature 4241032

[14] Steinle-Neumann G, Stixrude L, Cohen R E and Gülseren O 2001 Nature 41357

[15] Stixrude L, Cohen R E and Singh D J 1994 Phys. Rev. B 506442

[16] Stixrude L and Cohen R E 1995 Geophys. Res. Lett. 22125

[17] Wasserman E, Stixrude L and Cohen R E 1996 Phys. Rev. B 538296

[18] Gannarelli C M S, Alfe D and Gillan M J 2003 Phys. Earth Planet Inter. 139243

[19] Gannarelli C M S, Alfe D and Gillan M J 2005 Phys. Earth Planet Inter. 15267

[20] Gulseren O and Cohen R E 2002 Phys. Rev. B 65064103

[21] Hirshfelder J O, Curtiss C F and Bird R B 1954 Molecular Theory of Gases and Liquids (New York: Wiley)

[22] Sha X and Cohen R E 2006 Phys. Rev. B 74064103

[23] Wallace D C 1998 Phys. Rev. B 5815433

[24] Godwal B K, Modak P and Rao R S 2003 Solid State Commun. 125401

[25] Mao H K et al 2001 Science 292914

[26] DeWijs G A, Kresse G, Vocãdlo L, Dobson D, Alfe D, Gillan M J and Price G D 1998 Nature 392805

[27] Sha X and Cohen R E 2006 Phys. Rev. B 73104303

[28] Modak P, Verma A K, Rao R S, Godwal B K and Jeanloz R 2006 J. Mater. Sci. 411523

[29] Baroni S, de Gironcoli S, Corso A D and Giannozzi P 2001 Rev. Mod. Phys. 73515

[30] Merkel S, Goncharov A F, Mao H K, Gillet P and Hemley R J 2000 Science 2881626

[31] Steinle-Neumann G, Stixrude L and Cohen R E 2004 Proc. Natl Acad. Sci. USA $10133-6$

[32] Alfe D, Price G D and Gillan M J 2001 Phys. Rev. B 64045123

[33] Narasimhan S and de Gironcoli S 2002 Phys. Rev. B 65064302

[34] Nguyen J H and Holmes N C 2004 Nature 427339

[35] Rao R S, Modak P, Godwal B K and Sikka S K 1999 Phys. Rev. B 5913498

[36] Boehler R 1993 Nature 363534

[37] Shen G, Mao H K, Hemley R J, Duffy T S and Rivers M L 1998 Geophys. Res. Lett. 25373

[38] Williams Q, Jeanloz R, Bass J, Svendsen B and Ahrens T J 1987 Science 236181 